\title{
Cytogenetic basis of thelytoky in Apis mellifera capensis
}

\author{
Miles P. Cole-Clark ${ }^{1}$, Deborah A. Barton ${ }^{1}$, Michael H. Allsopp ${ }^{2}$, \\ Madeleine BeEkman ${ }^{1}$, Rosalyn S. Gloag ${ }^{1}$, Theresa C. Wossler ${ }^{3}$, Isobel RonaI ${ }^{1}$, \\ Nicholas SMIth ${ }^{1}$, Rebecca J. ReID ${ }^{1}$, Benjamin P. OLdROYD ${ }^{1}$ \\ ${ }^{1}$ Behaviour and Genetics of Social Insects Laboratory, University of Sydney, Macleay Building A12, Camperdown, \\ NSW 2006, Australia \\ ${ }^{2}$ Honey Bee Research Section, ARC-Plant Protection Research Institute, Private Bag X5017, Stellenbosch, Western Cape \\ 7599, South Africa \\ ${ }^{3}$ Department of Botany and Zoology, Centre for Invasion Biology, Stellenbosch University, P.B. X1 Matieland, \\ Stellenbosch, Western Cape 7602, South Africa
}

Received 23 August 2016 - Revised 8 February 2017 - Accepted 10 March 2017

\begin{abstract}
Haplodiploid insects reproduce both sexually and asexually; haploid males arise from unfertilized eggs, while diploid females arise from fertilized eggs. Some species can also produce female offspring by thelytokous parthenogenesis. For example, queenless workers of the Cape honey bee, Apis mellifera capensis, of South Africa can produce diploid female offspring from unfertilized eggs. Genetic evidence suggests that in A. m. capensis, diploidy is restored in zygotes by the fusion of two maternal pronuclei, the haploid descendants of the two alternate products of meiosis I. Here, we confirm this genetic evidence by direct cytological observation of pronucleus fusion. We also provide a description of how the fusion occurs at 4.5-5 h post oviposition and describe the meiotic events that lead up to and follow the fusion. Finally, we document numerous departures from the typical meiotic patterns, which likely explain some of the anomalous $A . m$. capensis individuals that have been previously identified genetically.
\end{abstract}

\section{Apis mellifera / thelytoky / central fusion / confocal fluorescence microscopy / haplodiploidy}

\section{INTRODUCTION}

There are approximately 200,000 described species in the insect order Hymenoptera, (ants, bees, wasps, and sawflies) (Leach et al. 2009). All Hymenoptera are haplodiploid; males are haploid and females are diploid. Haploid males arise via arrhenotokous parthenogenesis from unfertilized eggs, while diploid females are normally produced sexually (Heimpel and de Boer 2008).

Electronic supplementary material The online version of this article (doi:10.1007/s13592-017-0505-7) contains supplementary material, which is available to authorized users.

Corresponding author: B. Oldroyd, Benjamin.Oldroyd@sydney.edu.au Manuscript Editor: Klaus Hartfelder
However, in over 250 species of Hymenoptera identified thus far, diploid female offspring can also be produced asexually via thelytokous parthenogenesis (Engelstädter 2008; Rabeling and Kronauer 2013). In these species, females are produced parthenogenetically under particular circumstances. For example, little fire ant (Wasmannia auropunctata) workers are produced sexually, allowing colonies to exploit the benefits of genetic diversity among workers (Oldroyd and Fewell 2007). Queens, however, are produced asexually and are thus genetically "reincarnated" (Fournier et al. 2005).

The Cape honey bee, Apis mellifera capensis (hereafter "Capensis") has emerged as a key study species for understanding thelytokous parthenogenesis in haplodiploid organisms. Capensis is restricted to the Western Cape of South Africa 


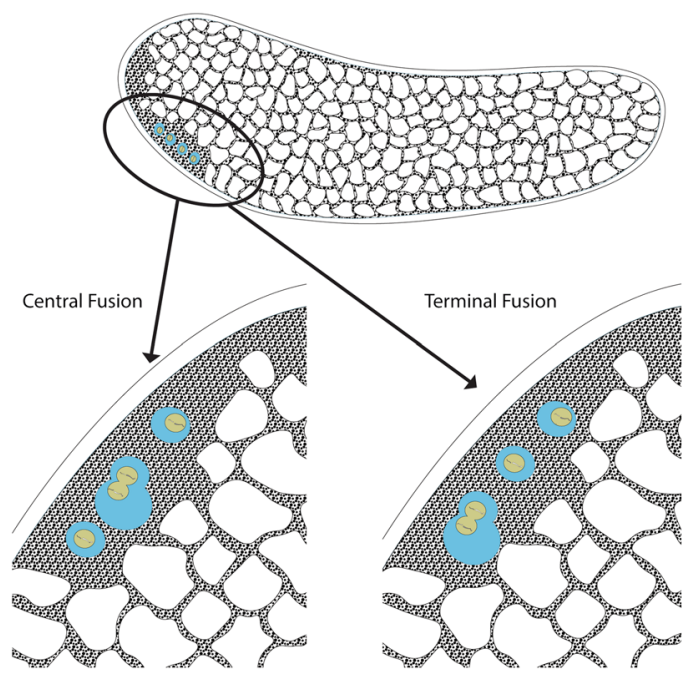

Figure 1. Expected alignment of pronuclei during central fusion and terminal fusion.

(Beekman et al. 2008; Oldroyd et al. 2008). Under normal circumstances, Capensis behaves as a typical haplodiploid social insect. The queen dominates reproduction, and workers rarely lay eggs (Beekman et al. 2009). Males arise from unfertilized queen-laid eggs, whereas females (daughter queens and workers) typically arise from fertilized eggs. When a colony has lost its queen and failed

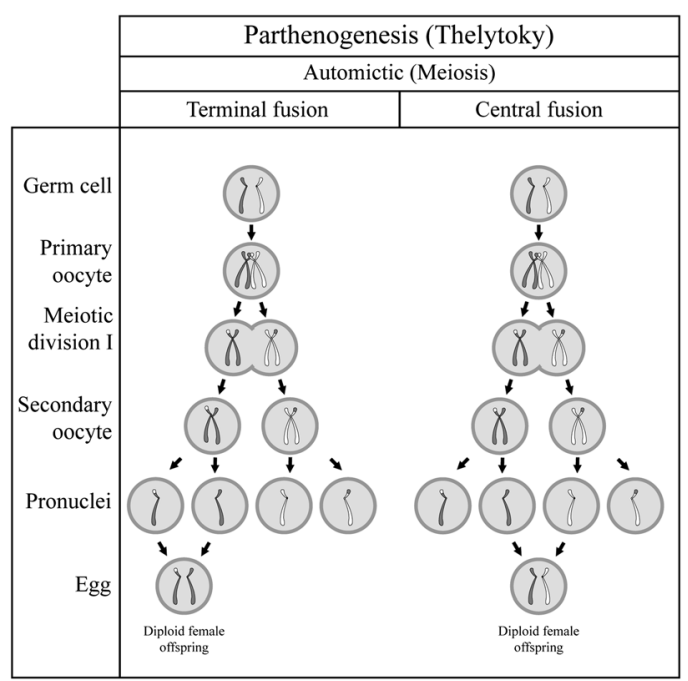

Figure 2. Comparison of meiotic divisions in different fusion mechanisms of parthenogenesis. The origin of the two pronuclei that fuse determines the type of automixis.
Figure 3. Meiotic events observed in worker-laid eggs of Apis mellifera capensis $0-4.5 \mathrm{~h}$ post oviposition. a Anaphase I (0-30 min); two groups of chromosomes during their separation. b Telophase I (30-60 min); complete separation of chromosomes with clustering at the polar ends of the now-separated pronuclei. c Prophase II (1-2 h); chromosomes are condensed. d Transitional stage between prophase II and metaphase II (2-3 h); chromosomes are condensed and lining up along the equator of each pronucleus. e Telophase II (4-4 $1 \frac{1}{2} \mathrm{~h}$ ); complete separation of the chromosomes resulting in the formation for four haploid pronuclei. $c$ chromosomes, $\mathrm{cr}$ chromatin thread. All images are twodimensional projections of three-dimensional confocal z-stacks.

to raise a new one, the workers begin to lay unfertilized eggs, as they do in all other honey bee species and subspecies (Winston 1991; Oldroyd and Wongsiri 2009). However when Capensis workers lay eggs, they usually develop as diploid females (Anderson 1963) via thelytoky (Verma and Ruttner 1983; Goudie and Oldroyd 2014). Up to half of all queens are the thelytokous offspring of workers, which arise from workerlaid eggs laid in queen cells (Jordan et al. 2008; Allsopp et al. 2010; Holmes et al. 2010; Moritz et al. 2011).

Honey bee eggs are laid with the maternal oocyte arrested in metaphase I (Sasaki and Obara 2002). Immediately following oviposition, the oocyte completes the divisions of meiosis to produce four haploid pronuclei (Verma and Ruttner 1983). Three of the pronuclei are then lost as polar bodies (Snodgrass 1956). If a sperm nucleus is present, it fuses with the remaining maternal pronucleus to form a zygote that will develop as a female. If the egg has not been fertilized, the remaining pronucleus begins to divide mitotically and will produce a male (Tucker 1958). In contrast to other honey bee subspecies, in the thelytokous eggs of Capensis workers, two of the four pronuclei often combine, as if one acted as a sperm, restoring diploidy and producing a female that is a partial clone of its mother. However, Goudie et al. (2012) reported that a minority of eggs that are laid by Capensis workers showed no evidence of genetic recombination and were therefore potentially produced by an ameiotic process. 

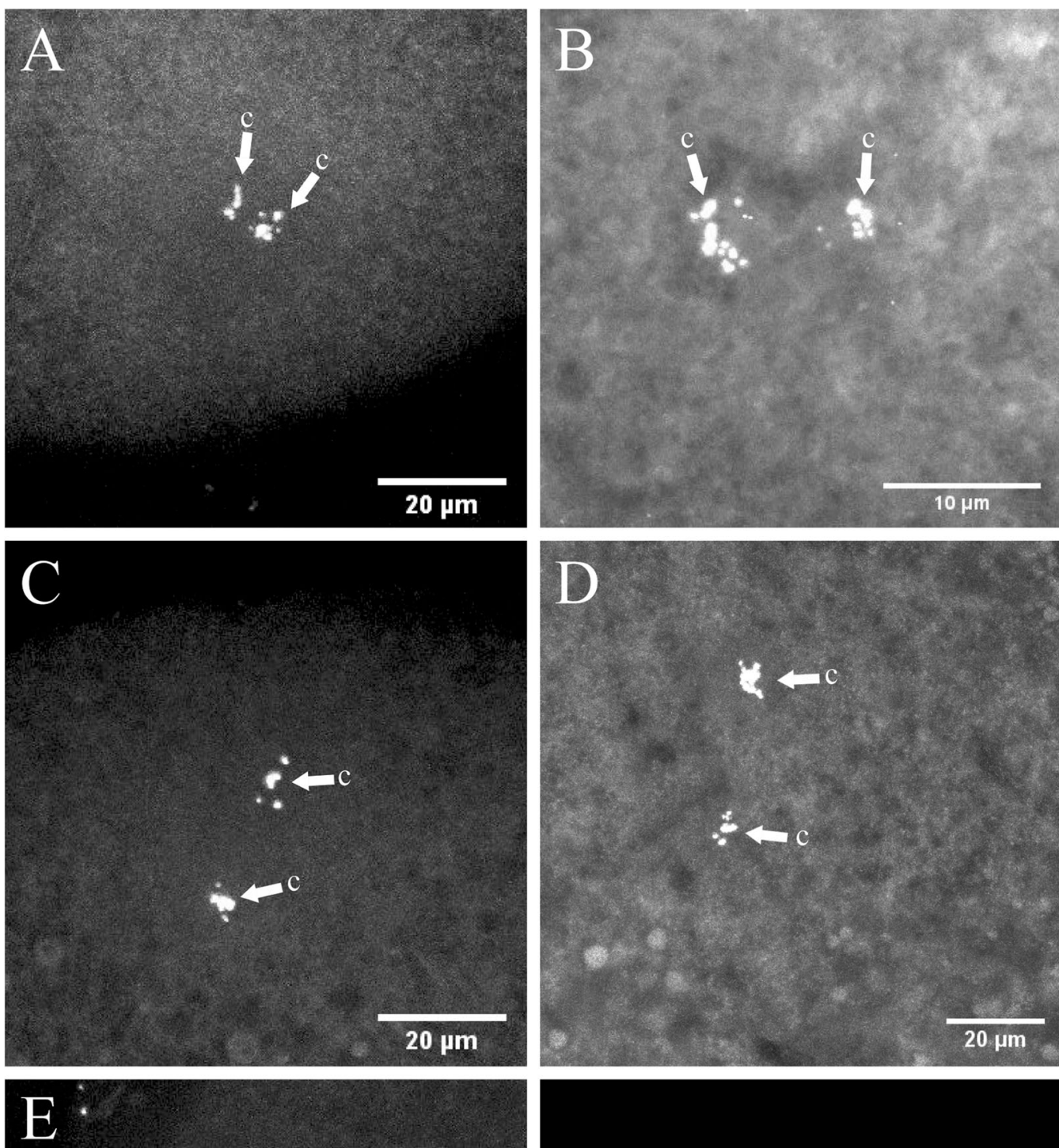

D

$$
\begin{aligned}
& 4<c \\
& 8<c
\end{aligned}
$$



s.

$$
k_{t \rightarrow c}
$$



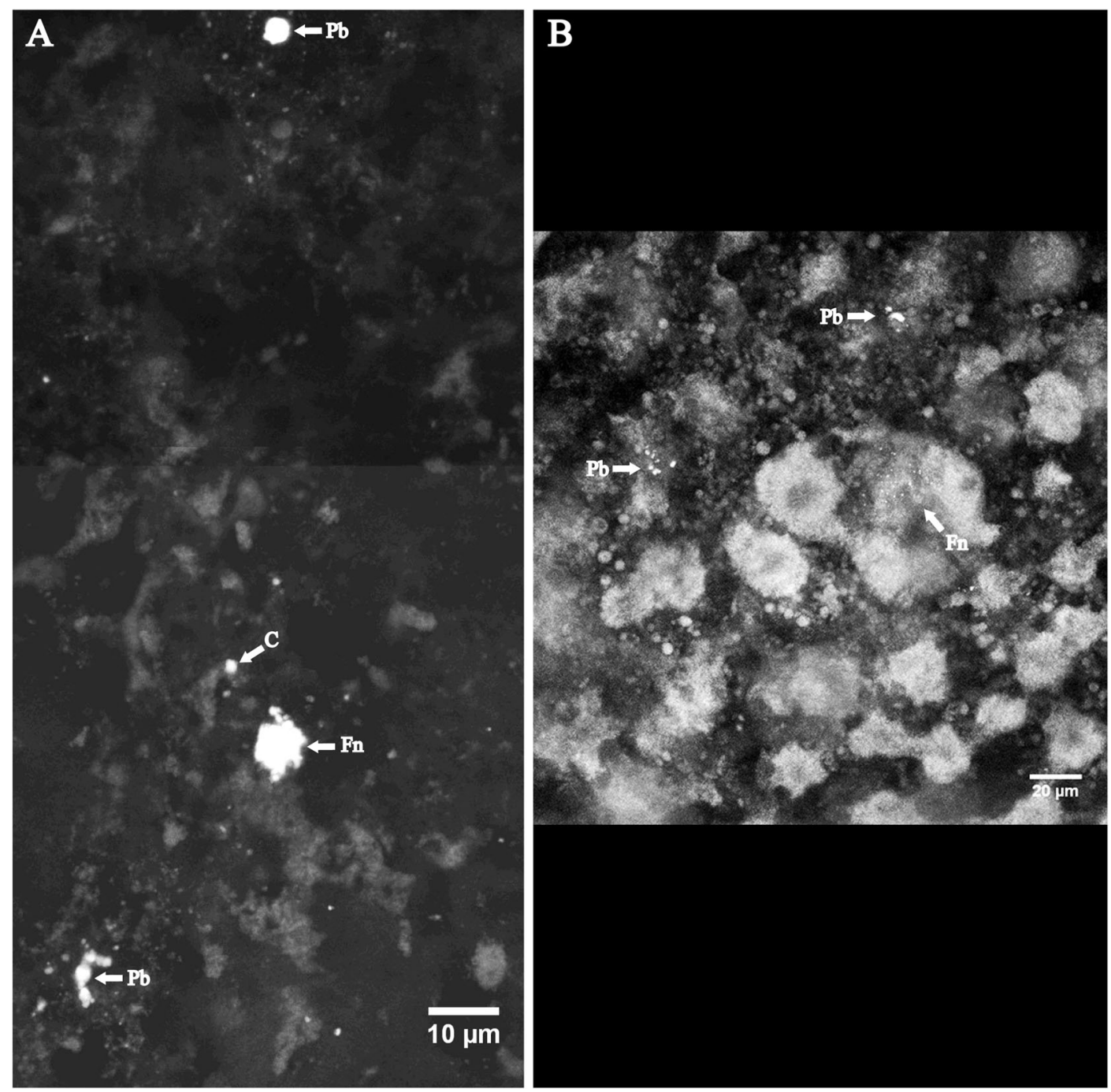

Figure 4. Central fusion of pronuclei in A. $m$. capensis worker-laid eggs. a The diploid fusion nucleus $(F n)$ with condensed chromosomes is at the center of the image. The two polar bodies $(\mathrm{Pb})$ (degrading pronuclei) are at the top and bottom of the image. The bright spot closest to the fusion nucleus $(c)$ appears to be a lagging chromosome. $\mathbf{b}$ Two degrading polar bodies $(\mathrm{Pb})$ adjacent to a single diploid nucleus $(\mathrm{Fn})$ that has moved towards the middle of the egg.

The genetic outcome of thelytokous fusion depends on which of the four pronuclei fuse (Fig. 1) and the extent of recombination. The two secondary oocytes, the products of meiosis I, both divide to produce two pronuclei (Fig. 1). Without recombination, the two daughter pronuclei of each secondary oocyte are identical. If the two pronuclei that fuse are derived from the same secondary oocyte (terminal fusion-Fig. 2), half of the genetic material of the mother is lost in the female offspring, and the individual will be homozygous at all loci (Pearcy et al. 2006; Goudie et al. 2012; Goudie and Oldroyd 2014). This kind of fusion is likely to be functionally lethal in honey bees because of the requirement of heterozygosity at the sex locus for normal female development (Beye et al. 2003). If pronuclei derived from alternate secondary oocytes fuse (central fusion-Fig. 2), then the resulting female offspring will be an exact clone of her mother (Goudie et al. 2012). There is also the possibility of random fusion, 
which will produce the expected outcomes of central and terminal fusion in equal proportion (AdachiHagimori et al. 2008).

Genetic recombination during meiosis I shuffles alleles between the four haploid products of meiosis II (Fig. 2). If recombination has been extensive at meiosis I (typically there are five or more crossover events per chromosome, Wallberg et al. 2015), alleles become shuffled between the four pronuclei (Goudie and Oldroyd 2014) and the genetic outcomes of terminal and central fusion become similar. Loci that are unlinked to the centromere and are heterozygous in the mother will become homozygous in the daughter one third of the time, and the mother's genotype will be retained two thirds of the time (Pearcy et al. 2006; Goudie et al. 2015). Observations of high levels of heterozygosity in thelytokous Capensis workers have been interpreted as evidence that the fusion is central, with reduced recombination (Moritz and Haberl 1994; Baudry et al. 2004). However, it is now thought that the frequency of recombination is only slightly lower overall than the normal honey bee meiosis (Goudie et al. 2012, 2014). Individuals that become homozygous as a result of recombination often have lethal allelic combinations and are removed from the population by selection (Goudie et al. 2014). By this means, heterozygosity is maintained in surviving individuals over many generations (Goudie et al. 2012).

Although genetic evidence suggests that thelytokous reproduction in Capensis is by central fusion (Oldroyd et al. 2008), there are odd exceptions. Although haploid males are typically rare in the progeny of Capensis workers, about $15 \%$ of the progeny of a clonal lineage of Capensis workers are haploid males (Goudie et al. 2015). In addition, strange cytogenetic events sometimes occur even in "normal" (arrhenotokous) Apis mellifera. For example, Rothenbuhler et al. (1952) demonstrated that diploid females can arise from the fusion of two sperm nuclei. In some honey bee strains, it is common for an embryo to develop from a zygote (the union of an egg and sperm) and from a secondary sperm cell. Adults arising from such eggs are gynandromorphs with some diploid (female) tissue and some haploid (male) tissue (Rothenbuhler et al. 1952; Tucker 1958; Narita et al. 2010).
The only cytological description of thelytokous parthenogenesis in the eggs of Capensis workers was conducted over 3 decades ago (Verma and Ruttner 1983). Here, we use confocal fluorescence microscopy to systematically describe thelytokous egg development, from oviposition to blastoderm formation. This technique allows visualization of chromosomes in three dimensions. We provide evidence of central fusion and identify several abnormal processes that likely explain why genetic data are not always consistent with central fusion.

\section{MATERIALS AND METHODS}

\subsection{Specimen collection}

We collected worker-laid eggs from queenless A. m. capensis colonies at the Agricultural Research Council Plant Protection Institute (ARC PPRI) in Stellenbosch, South Africa, between December 2015 and January 2016. Queenless Capensis workers often construct queen cells into which they lay thelytokous eggs. We removed any eggs present in queen cells and then collected worker-laid eggs at 30-min intervals for up to $6 \mathrm{~h}$.

We transferred eggs in the field into Eppendorf tubes containing a fixative solution (5\% paraformaldehyde, $100 \mathrm{mM}$ PIPES, $2 \mathrm{mM} \mathrm{MgSO}_{4}$, $10 \mathrm{mM} \mathrm{CaCl}_{2}$ in $100 \%$ heptane) adapted from Oxley et al. (2014) and Dearden et al. (2009). Eggs were fixed for $60 \mathrm{~min}$ at room temperature. We then rinsed the eggs three times for 5 min per rinse in a solution of $100 \mathrm{mM}$ PIPES, $2 \mathrm{mM}$ $\mathrm{MgSO}_{4}$, and $1 \mathrm{mM}$ EGTA. Fixed eggs were then stained immediately or placed in methanol $(100 \%$ $v / v \mathrm{MeOH})$ (Dearden et al. 2009) and frozen $\left(-20^{\circ} \mathrm{C}\right)$ for storage.

\subsection{Egg staining and imaging}

We rehydrated the stored eggs with a decreasing concentration of methanol in $0.1 \mathrm{M}$ phosphate buffer solution ( $\mathrm{pH} 7.4)(75,50,25$, and $0 \% v / v)$ for $20 \mathrm{~min}$ per solution.

We stained the eggs with SYTOX Blue Nucleic Acid Stain (molecular probes) or propidium iodide (PI) (Sigma-Aldrich) in a 1:1000 dilution for $60 \mathrm{~min}$. We then rinsed excess stain from the eggs with three 


Figure 5. Meiotic abnormalities observed in workerlaid eggs of A. m. capensis . a Chromosomes (c) undergoing anaphase I with apparent lagging chromosomes $(l c)$. b Chromosomes $(c)$ at the telophase I stage of development with 2-3 lagging chromosomes $(l c)$. c Possible haploid egg development; a single haploid pronucleus is present towards the center of egg $(c)$ and appears ready for further development. The remaining pronuclei $(c)$ can be seen at the bottom of the image in close proximity to each other. The other fluorescing structures in this image are likely to be overstained yolk globules. d An anucleate egg; the periplasm (outlined by the white line ) is devoid of any genetic material. The chorion (ch) is clearly visible surrounding the egg. e Egg showing multiple sites of chromosome groups. f Egg with one set of chromosomes $(c)$ located at the edge of the periplasm and a more developed nucleus formed centrally in the periplasm $(c)$. Because the central pronucleus is well developed, we expect the others to be degraded by this stage if development was normal. Chorion $(\mathrm{ch})$ is also visible.

5-min rinses in Milli-Q water. Eggs stained with PI were first digested with $1 \mathrm{mg} / \mathrm{mL}$ RNAse for $60 \mathrm{~min}$ in order to reduce background fluorescence (Suzuki et al. 1997) and then rinsed with Milli-Q water. We mounted eggs in $70 \%(v / v)$ glycerol solution on a glass slide, covered them with a cover slip, and sealed the preparation with clear nail polish.

\subsection{Imaging}

We examined eggs stained with SYTOX at the University of Stellenbosch with a Zeiss LSM 780 ELYRA S1A confocal microscope, fluoresced using an argon laser line at $458 \mathrm{~nm}$. We captured images with a GaSsP detector $32+2$ PMT and transmitted light detector T-PMT.

We examined eggs at the University of Sydney, after staining with PI, with a $543 \mathrm{~nm}$ HeNe laser mounted to a Zeiss LSM 5 PASCAL confocal microscope. We used objectives Plan-Neofluar $10 \times / 0.30$, Archoplan 40×/0.80, Archoplan $63 \times 1$ 0.95 , and Plan-Neofluar $100 \times / 1.30$ oil.

We initially processed images using Carl Zeiss imaging software (Zen blue on the Zeiss LSM 78 ELYRA S1 and LSM 5 v4.2 on the Zeiss LSM 5 PASCAL) and further processed the images using ImageJ, Adobe Photoshop, and Adobe Illustrator.

\section{RESULTS}

\subsection{Meiotic events prior to nuclear fusion}

In Figs. 3 and 4, we present montages of typical eggs in the various stages of meiosis and nuclear fusion, respectively, in which chromosomes have been fluorescently stained. These images are necessarily quite small and are projected in only two dimensions, so we provide full-sized versions of the same images (Figs. S1, S2, S3, S4, S5, S6, S7, S8, S9, S10, S11, S12, $\mathrm{S} 13$ ) and animations of the individual planes of each image (Movies M1, M2, M3, M4, M5, M6, M7, M8, M9, M10, M11, M12, M13) in supplementary material. The animations in particular give a sense of the meiotic events as they occur in three dimensions.

We observed the full range of meiotic events across the 169 eggs imaged. All divisions occurred within the anterior or anteroventral end of the eggs within the periplasm. Anaphase I was characterized by separation of chromosomes towards either pole ( $n=2$ imaged eggs; Fig. 3a, S1, and Movie M1). Telophase I at 30-60 min after oviposition ( $n=10$ imaged eggs) showed chromosomes in two distinct groups (Fig. 3b, S2, and Movie M2) suggestive of the two secondary oocytes.

We observed prophase II in eggs collected $1-2 \mathrm{~h}$ post oviposition ( $n=2$ imaged eggs), whereby two separate concentrations of condensed chromosomes were visible (Fig. 3c, S3, and Movie M3).

A transitional stage between prophase II and metaphase II ( $n=3$ imaged eggs) can be seen in Fig. 3d, S4, and Movie M4. The chromosomes are condensed to the same degree as those in (Fig. 3c) and S3 but are more strongly aligned along the equatorial plane of the pronuclei.

Complete separation of the chromosomes into four haploid pronuclei is clearly visible in the image of telophase II (Fig. 3e, S5, and Movie M5).

Autofluorescent yolk material was present at all stages of meiosis, as was the tough outer chorion up until blastoderm layer formation. There was no evidence of nuclear membranes in any image suggesting that they do not form in early honey bee embryos or did not stain. 


\subsection{Central versus terminal fusion}

We observed two instances of central fusion in worker-laid eggs that were collected 4.5 to $5 \mathrm{~h}$ post oviposition. Figure 4a, S6, and Movie M6 clearly show three large chromosome clusters within the egg, with the central cluster, which we interpret as the fusion nucleus, roughly twice the size of the chromosomal groups on either side, which we interpret as the polar bodies. The chromosomes in the centrally fused nucleus are condensed and positioned towards the central axis of the egg with one or possibly a pair of chromosomes lagging. The top polar body is also condensed, but appears to be breaking down (Movie M6). The polar body at the bottom of Fig. 4a and S7 also appears to be in the process of degeneration as the chromosomes are diffuse and very unlike the highly condensed chromosomes of the fusion nucleus.

Figure 4b, S7, and Movie M7 show a diploid fusion nucleus positioned towards the center of the egg, with what we infer to be the haploid pronuclei at the top and bottom regions of the image.

We did not observe evidence of terminal fusion in any of the 169 eggs successfully imaged.

\subsection{Meiotic abnormalities}

Chromosome lag is associated with aneuploidy. We observed lagging chromosomes in five eggs. Figure 5a, S8, and Movie M8 depict chromosome lag at anaphase I. Two distinctly separate lines of chromosomes indicative of anaphase I can be seen with single chromosomes staggered in between. Similarly, we observed chromosome lag at telophase I (Fig. 5b, S9, and Movie M9). Here, two to three single chromosomes are positioned between the two nearly separated secondary oocytes.

We observed haploid development with a single haploid pronucleus moving towards the center of the egg for further division in five eggs (Fig. 5c, S10, and Movie M10).

We imaged other meiotic oddities including eggs with unusual groupings of chromosomes. For example, Fig. 5e, S12, and Movie M12 show five groups of chromosomes in close proximity in the periplasm. The egg in Fig. 5f, S13, and Movie M13 has a distinctly formed nucleus within the periplasm at the anterior of the egg, as well as a set of chromosomes towards the outside edge of the egg. This egg is abnormal because by this stage of the formation of the fusion nucleus, the chromosomes present towards the outside of the egg should have disappeared as polar bodies.

Interestingly, $18 \%$ of imaged eggs lacked nucleic acid and were therefore anucleate (Fig. 5d, S11, and Movie M11). These anucleate eggs were laid at the same time as other eggs that had developed a blastoderm layer. As such they should also have had a blastoderm layer but they apparently lacked sufficient genetic material to be viable.

\section{DISCUSSION}

This study provides cytological evidence that central fusion occurs during thelytokous egg development in Capensis worker eggs. In particular, the central fusion shown in Fig. 4 shows the fusion nucleus situated between the two polar bodies, confirming inferences from genetic (Moritz and Haberl 1994; Goudie et al. 2015) and early cytogenetic (Tucker 1958; Verma and Ruttner 1983) studies. In contrast, we found no evidence of terminal fusion, which would manifest as a single diploid fusion nucleus and a pair of degenerating haploid nuclei positioned adjacent to the fusion nucleus (Suomalainen et al. 1987). Our findings therefore support, extend, and improve earlier cytogenetic studies. However, because honey bee chromosomes are so small, current confocal microscopy methods are at the limit of clear resolution, and we therefore acknowledge that our interpretations are tentative.

We observed the majority of meiotic chromosomal events. Our study suggests that the period during which nuclear fusion events occur is brief - of the order of $30 \mathrm{~min}$ - most likely 4 to $4.5 \mathrm{~h}$ post oviposition, and the whole meiotic process is complete in $6 \mathrm{~h}$. (See Fig. 6 for a summary of what we believe is the time course of a thelytokous meiosis in the Capensis workerlaid egg). Given the brief period during which fusion occurs, the limited control over when workers lay eggs in the field and the relatively 




Figure 6. Timeline of meiotic divisions in capensis worker-laid eggs from anaphase I to complete blastoderm layer formation.

high frequency of eggs where no fusion event occurs because the eggs are either haploid (3\%) or anucleate $(18 \%)$, it is difficult to image eggs at the precise moment of fusion. Therefore, we cannot exclude the possibility that terminal fusion also occurs in A. m. capensis at low frequency.

Sasaki and Obara (2002) showed that honey bee eggs are laid arrested in metaphase I and proceed to anaphase I within 15 min of oviposition. Eggs require mechanical squeezing in the oviduct, in order to proceed to anaphase. This is consistent with our observations of telophase I 30-60 min after oviposition. Sasaki and Obara also showed that eggs are only receptive to sperm within a few minutes of mechanical squeezing and require both squeezing and sperm to initiate diploid embryogenesis. It is interesting to note that under thelytoky, pronucleus fusion occurs some $4 \mathrm{~h}$ after the usual time for fertilization, suggesting that the two processes are nonhomologous.
We saw no evidence of nuclear membranes in any image, which we interpret as an absence of staining. However, fusion of pronuclei presumably requires that the nuclear membranes disintegrate, and so perhaps the nuclear membrane is indeed absent throughout the second division meiosis of thelytokous parthenogenesis.

We observed chromosome lag in anaphase I and telophase I on four occasions. Lagging chromosomes have been previously observed in unfertilized eggs that are destined for development as haploid males (Kumbkarni 1965), so this may indicate that these eggs were developing arrhenotokously, not thelytokously. We also observed that $3 \%$ of imaged eggs appeared to be haploid based on an individual pronucleus moving towards the center of the egg without evidence of central or terminal fusion. The frequency of haploid eggs in Capensis worker progeny varies from $0.65 \%$ (Goudie et al. 
2015 ) to $15 \%$ (Goudie et al. 2012) depending on the population.

The high frequency of anucleate eggs is of particular interest, as such eggs predispose androgenesis: the development of sperm nuclei into embryos and beyond without the benefit of a maternal genome (Schwander and Oldroyd 2016). In haplodiploids, there are no developmental impediments to a sperm cell dividing within an anucleate egg and producing a male, a clone of his father (Schwander and Oldroyd 2016). Androgenesis is documented in the ants Wasmannia auropunctata (Fournier et al. 2005; Foucaud et al. 2010), Vollenhovia emeryi (Ohkawara et al. 2006; Kobayashi et al. 2008), Cardiocondyla kagutsuchi (Okita and Tsuchida 2016), Paratrechina longicornis (Pearcy et al. 2011), and in some strains of honey bee (Koeniger et al. 1989). Although we have no evidence of androgenesis in Capensis, the possibility now needs to be front of mind when considering the genotypes of male offspring of instrumentally inseminated Capensis queens, which may sometimes lay anucleate eggs, allowing the clonal development of a male from a sperm that has penetrated the egg.

In addition to the possibility of androgenesis, the fluidity of cytogenetic events in early Capensis eggs and embryos may also facilitate the development of gynandromorphs, mosaics of male and female tissue arising from the development of a diploid zygote and an accessory haploid sperm cell (Rothenbuhler et al. 1949), or even zoogenesis, in which two sperm cells fuse to make a diploid zygote (Rothenbuhler et al. 1952).

Our findings indicate that eggs $4.5-5.0 \mathrm{~h}$ post oviposition are needed in order to image pronuclear fusion. By sampling large numbers of eggs during this time window, it should be possible to quantify the frequency of arrhenotoky, thelytoky, and anucleate eggs. In addition, if terminal fusion occurs at low frequency, it may be possible to observe this directly. Based on genetic evidence, Goudie et al. (2012) suggested that some Capensis eggs may be produced ameiotically. Cytogenetic confirmation of ameiotic reproduction would be very useful. Nonrecombinant meiosis is likely to have nongenetic causes for it would otherwise rapidly go to fixation, since it does not involve the loss of heterozygosity that is normally associated with thelytoky (Goudie et al. 2012).

\section{ACKNOWLEDGEMENTS}

We thank Ms. Lize Engelbrecht for the use of the CAF fluorescent microscopy unit at the Stellenbosch University. This work was funded by the Australian Research Council project DP15101985 to B. Oldroyd and R. Gloag.

\section{Authors' contributions}

MPC-C, DAB, and BPO performed research. MPC-C, DB and BPO wrote the paper; MHA and TCW provided materials and resources; $\mathrm{MB}, \mathrm{NS}$, and RR contributed to field work; RSG contributed to writing.

\section{OPEN ACCESS}

This article is distributed under the terms of the Creative Commons Attribution 4.0 International License (http://creativecommons.org/licenses/by/4.0/), which permits unrestricted use, distribution, and reproduction in any medium, provided you give appropriate credit to the original author(s) and the source, provide a link to the Creative Commons license, and indicate if changes were made.

Base cytogénétique de la thélytoquie chez Apis mellifera capensis

Apidae / fusion centrale / microscopie confocale de fluorescence / haplodiploïdie

Zytogenetische Basis der Thelytoky bei Apis mellifera capensis

Apis mellifera / Thelytokie / zentrale Fusion / konfokale Fluoreszenzmikroskopie / Haplodiploidie

\section{REFERENCES}

Adachi-Hagimori T., K. Miura, R. Stouthamer (2008) A new cytogenetic mechanism for bacterial endosymbiontinduced parthenogenesis in Hymenoptera. Proc R. Soc. Lond. B 275, 2667-2673.

Allsopp M.H., M. Beekman, R.S. Gloag, B.P. Oldroyd (2010) Maternity of replacement queens in the 
thelytokous Cape honey bee Apis mellifera capensis . Behav. Ecol. Sociobiol. 64, 567-574.

Anderson R. (1963) The laying worker in the Cape honeybee, Apis mellifera capensis. J. Apic. Res. 2, 85-92.

Baudry E., P. Kryger, M. Allsopp, N. Koeniger, D. Vautrin, F. Mougel, J.-M. Cornuet, M. Solignac (2004) Whole genome scan in thelytokous-laying workers of the Cape honey bee (Apis mellifera capensis): central fusion, reduced recombination rates and centromere mapping using half-tetrad analysis. Genetics 167, 243-252.

Beekman M., M. Allsopp, T.C. Wossler, B.P. Oldroyd (2008) Factors affecting the dynamics of the honey bee (Apis mellifera) hybrid zone of South Africa. Heredity 100, 13-18.

Beekman M., M.H. Allsopp, L.A. Jordan, J. Lim, B.P. Oldroyd (2009) A quantitative study of worker reproduction in queenright colonies of the Cape honey bee, Apis mellifera capensis . Mol Ecol 18, 2722-2727.

Beye M., M. Hasselmann, M.K. Fondrk, R.E. Page, S.W. Omholt (2003) The gene $c s d$ is the primary signal for sexual development in the honeybee and encodes an SR-type protein. Cell 114, 419-429.

Dearden P.K., E.J. Duncan, M.J. Wilson (2009) Fixation and storage of honeybee (Apis mellifera) tissues. Cold Spring Harbor Protocols 4, doi:10.1101/pdb. prot5224.

Engelstädter J. (2008) Constraints on the evolution of asexual reproduction. BioEssays 30, 1138-1150.

Foucaud J., A. Estoup, A. Loiseau, O. Rey, J. Orivel (2010) Thelytokous parthenogenesis, male clonality and genetic caste determination in the little fire ant: new evidence and insights from the lab. Heredity 105, 205-212.

Fournier D., A. Estoup, J. Orivel, J. Foucaud, H. Jourdan, J. Le Breton, L. Keller (2005) Clonal reproduction by males and females in the little fire ant. Nature 435, $1230-1234$.

Goudie F., B.P. Oldroyd (2014) Thelytoky in the honey bee, Apidologie 45, 306-326.

Goudie F., M.H. Allsopp, M. Beekman, P.R. Oxley, J. Lim, B.P. Oldroyd (2012) Maintenance and loss of heterozygosity in a thelytokous lineage of honey bees (Apis mellifera capensis ). Evolution 66, 1897-1906.

Goudie F., M.H. Allsopp, B.P. Oldroyd (2014) Selection on overdominant genes maintains heterozygosity along multiple chromosomes in a clonal lineage of honey bee. Evolution 68, 125-136.

Goudie F., M.H. Allsopp, M. Solignac, M. Beekman, B.P. Oldroyd (2015) The frequency of arrhenotoky in the normally thelytokous Apis mellifera capensis worker and the Clone reproductive parasite. Insectes Soc. 62, 352-333.

Heimpel G.E., J.G. de Boer (2008) Sex determination in the Hymenoptera. Annu. Rev. Ent. 53, 209-230.

Holmes M.J., B.P. Oldroyd, M.H. Allsopp, J. Lim, T.C. Wossler, M. Beekman (2010) Maternity of emergency queens in the Cape honey bee, Apis mellifera capensis . Mol. Ecol. 19, 2792-2799.

Jordan L.A., M.H. Allsopp, B.P. Oldroyd, T.C. Wossler, M. Beekman (2008) Cheating honeybee workers produce royal offspring. Proc R. Soc Lond B 275, 345-351.

Kobayashi K., E. Hasegawa, K. Ohkawara (2008) Clonal reproduction by males of the ant Vollenhovia emeryi (Wheeler), Entomol. Sci 11, 167-172.

Koeniger N., C. Hemmling, T. Yoshida (1989) Drones as sons of drones in Apis mellifera. Apidologie 20, 391394.

Kumbkarni C. (1965) Cytological Studies in Hymenoptera. Cytologia 30, 222-228.

Leach I.M., B.A. Pannebakker, M.V. Schneider, G. Driessen, L. Van de Zande, L.W. Beukeboom (2009) Thelytoky in Hymenoptera with Venturia canescens and Leptopilina clavipes as case studies, in: Schön I., Martens K. van Dijk P. (Eds.), Lost Sex, Springer, Dordrecht, pp. 347-375.

Moritz R.F., M. Haberl (1994) Lack of meiotic recombination in thelytokous parthenogenesis of laying workers of Apis mellifera capensis (the Cape honeybee). Heredity $73,98-102$.

Moritz R.F., H.M.G. Lattorff, K.L. Crous, R.H. Hepburn (2011) Social parasitism of queens and workers in the Cape honeybee (Apis mellifera capensis). Behav. Ecol. Sociobiol. 65, 735-740.

Narita S., R.A. Pereira, F. Kjellberg, D. Kageyama (2010) Gynandromorphs and intersexes: potential to understand the mechanism of sex determination in arthropods. Terr. Arthropod Rev. 3, 63-96.

Ohkawara K., M. Makayama, A. Satoh, A. Trindl, J. Heinze (2006) Clonal reproduction and genetic caste differences in a queen-polymorphic ant, Vollenhovia emeryi. Biol. Lett. 2, 359-363.

Okita, I., Tsuchida, K. (2016) Clonal reproduction with androgenesis and somatic recombination: the case of the ant Cardiocondyla kagutsuchi. The Science of Nature 103

Oldroyd B.P., J.H. Fewell (2007) Genetic diversity promotes homeostasis in insect colonies. Trends Ecol. Evol. 22, 408-413.

Oldroyd B.P., S. Wongsiri (2009) Asian honey bees: biology, conservation, and human interactions, Harvard University Press, Boston.

Oldroyd B.P., M.H. Allsopp, R.S. Gloag, J. Lim, L.A. Jordan, M. Beekman (2008) Thelytokous parthenogenesis in unmated queen honeybees (Apis mellifera capensis ): Central fusion and high recombination rates. Genetics 180, 359-366.

Oxley P.R., L. Ji, I. Fetter-Pruneda, S.K. McKenzie, C. Li, H.F. Hu, G.J. Zhang, D.J.C. Kronauer (2014) The genome of the clonal raider ant Cerapachys biroi, Curr. Biol. 24, 451-458.

Pearcy M., O. Hardy, S. Aron (2006) Thelytokous parthenogenesis and its consequences on inbreeding in an ant. Heredity 96, 377-382. 
Pearcy M., M.A.D. Goodisman, L. Keller (2011) Sib mating without inbreeding in the longhorn crazy ant. Proc. R. Soc. Lond. B 278, 2677-2681.

Rabeling C., D.J.C. Kronauer (2013) Thelytokous parthenogenesis in eusocial Hymenoptera. Annu. Rev. Ent. 58, 273-292.

Rothenbuhler W.C., J.W. Polhemus, J.W. Gowen, O.W. Park (1949) Gynandromorphic honey bees. J. Hered. 40, 308-311.

Rothenbuhler W.C., J.W. Gowen, O. Park (1952) Androgenesis with zygogenesis in gynandromorphic honeybees (Apis mellifera L. ). Science 115, 637-638.

Sasaki K., Y. Obara (2002) Egg activation and timing of sperm acceptance by an egg in honeybees (Apis mellifera L.). Insectes Soc. 49, 234-240.

Schwander, T., Oldroyd, B. (2016) Androgenesis: where males hijack eggs to clone themselves, Philos. Trans. R. Soc. B-Biol. Sci. 371, 20150534.
Snodgrass R., 1956. Anatomy of the honey bee. Cornell University Press, London.

Suomalainen E., A. Saura, J. Lokki (1987) Cytology and evolution in parthenogenesis. CRC Press, Florida.

Suzuki T., K. Fujikura, T. Higashiyama, K. Takata (1997) DNA staining for fluorescence and laser confocal microscopy. J. Histochem. Cytochem. 45, 49-53.

Tucker K.W. (1958) Automictic parthenogenesis in the honey bee. Genetics 43, 299.

Verma S., F. Ruttner (1983) Cytological analysis of thelytokous parthenogenesis in the Cape honey bee Apis mellifera capensis. Apidologie 14, 41-57.

Wallberg A., S. Glémin, M.T. Webster (2015) Extreme recombination frequencies shape genome variation and evolution in the honeybee, Apis mellifera. PLOS Genetics 11, e1005189.

Winston M.L. (1991) The biology of the honey bee, Harvard University Press, Boston. 